

\section{NOTICE}

This report was prepared as an account of work sponsored by an agency of the United States Government. Neither the United States Government nor any agency thereof, nor any of their employees, makes any warranty, express or implied, or assumes any legal liability or responsibility for the accuracy, completeness, or usefulness of any information, apparatus, product, or process disclosed, or represents that its use would not infringe privately owned rights. Reference herein to any specific commercial produce, process, or service by trade name, trademark, manufacturer, or otherwise, does not necessarily constitute or imply its endorsement, recommendation, or favoring by the United States Government or any agency thereof. The views and opinions of authors expressed herein do not necessarily state or reflect those of the United States Government or any agency thereof.

\section{NOTICE}

This report has been reproduced from the best available copy. Available in paper copy and microfiche.

Number of pages in this report: 9

DOE and DOE contractors can obtain copies of this report from:

Office of Scientific and Technical Information

P.O. Box 62

Oak Ridge, TN 37831;

(615) $576-8401$.

This report is publicly available from the:

National Technical Information Service

Department of Commerce

5285 Port Royal Road

Springfield, Virginia 22161

(703) $487-4650$ 


\section{DISCLAIMER}

Portions of this document may be illegible in electronic image products. Images are produced from the best available original document. 


\title{
Renormalized Dissipation in Plasmas with Finite Collisionality
}

\author{
Scott E. Parker ${ }^{1}$ and Daniele Carati ${ }^{2}$ \\ ${ }^{1}$ Princeton Plasma Physics Laboratory, Princeton University, P.O. Box 451, Princeton, NJ 08543 \\ ${ }^{2}$ Service de Physique Statistique, Plasma et Optique Nonlinéaire \\ Université Libre de Bruxelles, Campus Plaine C.P. 231, B-1050 Bruxelles, Belgium
}

(March 7, 1995)

\begin{abstract}
A nonlinear truncation procedure for Fourier-Hermite expansion of Boltzmann-type plasma equations is presented which eliminates fine velocity scale, taking into account its effect on coarser scales. The truncated system is then transformed back to $(x, v)$ space which results in a renormalized Boltzmann equation. The resulting equation may allow for coarser velocity space resolution in kinetic simulations while reducing to the original Boltzmann equation when fine velocity scales are resolved. To illustrate the procedure, renormalized equations are derived for one dimensional electrostatic plasmas in which collisions are modeled by the Lenard-Bernstein operator.
\end{abstract}

PACS: 52.25.DG, 52.35.Ra, 52.64.-y

It has long been known (see for example Refs. 1-10), that the Landau damping (or phase mixing) of an initial perturbed wave will take a spatial variation with a slow velocity variation and damp it spatially causing fine wiggles to appear in velocity space. This fine velocity scale eventually damps away due to collisions $[1,2,6,7,9,10]$. This concept has recently been discussed by Hammett and coworkers [10] as justification for why few moment fluid equations with added dissipative terms may be adequate for modeling kinetic effects. Drawing upon this idea, along with analogies with renormalization methods in Navier-Stokes (NS) fluid turbulence [11-13] we derive renormalized dissipative linear and nonlinear mode coupling terms in a Boltzmann-type equation. The renormalized equation can be used in kinetic simulations to reduce fine scale velocity space resolution and simply reduces to the original Boltzmann equation when fine velocity scale is resolved. Also, the truncation procedure described here requires no knowledge of the details of linear kinetic theory, and provides a way to calculate nonlinear corrections to Landau-Fluid [14] type closures.

To illustrate the renormalization procedure we will study a uniform one dimensional electrostatic plasma with kinetic electrons and fixed ions where collisions are modeled using the Lenard-Bernstein operator

$$
\partial_{t} f+v \partial_{x} f-\frac{e}{m_{e}} E \partial_{v} f=\nu_{0} \partial_{v}\left(v f+v_{t e}^{2} \partial_{v} f\right)
$$

along with Poisson's equation: $\partial_{x} E=4 \pi e\left(n_{0}-\int f d v\right)$, where $n_{0}$ is the ion density, $f=f(x, v, t)$ is the electron distribution function, $v_{t e}$ is the thermal velocity, and $\nu_{0}$ is the collision frequency. The calculations presented below can be generalized to more complex kinetic equations and this will be presented elsewhere.

For clarity, we begin with linearized equations (the nonlinear problem is discussed below), i.e. $f(x, v, t)=F(v)+$ $\delta f(x, v, t)$, keeping terms $O(\delta f)$, where $F(v)$ is assumed to be Maxwellian. It is most natural to use a Fourier-Hermite expansion of Eq. (1)

$$
f(x, v, t)=\int d \omega e^{-i \omega t} \sum_{k, \ell} a_{k}^{\ell}(\omega) e^{i k x} e^{-v^{2} / 2} h_{\ell}(v)
$$

where $h_{\ell}(v)$ are orthonormal Hermite polynomials. The resulting linearized equations for the $a_{k}^{\ell}$ 's are then given by

$$
\left(-i \omega+\nu_{0} \ell\right) a_{k}^{\ell}+i k\left\{\sqrt{\ell} a_{k}^{\ell-1}+\sqrt{\ell+1} a_{k}^{\ell+1}\right\}=0,
$$

for $\ell=2,3,4, \ldots$, while the first two equations are

$$
\begin{aligned}
& -i \omega a_{k}^{1}+i k\left\{a_{k}^{0}+\sqrt{2} a_{k}^{2}\right\}+\nu_{0} a_{k}^{1}+E_{k}=0 \\
& -i \omega a_{k}^{0}+i k a_{k}^{1}=0
\end{aligned}
$$


The Poisson equation becomes $E_{k}=i(2 \pi)^{1 / 4} a_{k}^{0} / k$. Dimensionless units are used so that the plasma frequency, the Debye length and the thermal velocity are all unity, $\left(\omega_{p e}=1, \lambda_{D e}=1, v_{t e}=\lambda_{D e} \omega_{p e}=1\right)$. Thus, $\nu_{0}=\nu_{0} / \omega_{p e}$ is the normalized collision frequency. Details can be found in Refs. $[1,2,4]$. A linear truncation of the discrete Fourier-Hermite expansion of the Vlasov equation using a Landau-fluid type closure has been developed by Smith and Hammett [15]. Here, we discuss a new truncation procedure which makes no reference to the plasma dispersion function and takes into account the effect of nonlinearities (which will be discussed later).

We are interested in truncating the system at large $\ell$ (which correspond to fine velocity scales), and need some starting point characterized by a "resolution" index $\ell_{r}$ with the property $a_{k}^{\ell} \approx 0$ if $\ell>\ell_{r}$. This index $\ell_{r}$ (which is estimated below) determines how many coefficients are needed to fully resolve the linear problem Using $a_{k}^{\ell_{r}+1} \approx 0$, the equation for $a_{k}^{\ell_{r}}$ becomes

$$
\left(-i \omega+\nu_{0} \ell_{r}\right) a_{k}^{\ell_{r}}+i k \sqrt{\ell_{r}} a_{k}^{\ell_{r}-1} \approx 0 .
$$

This relation is further simplified by considering the small frequency limit in which $\omega$ is neglected compared to $\nu_{0} \ell_{r}$ (this is referred to as the investigation of long time behaviors in renormalization group theories [11]). The solution for $a_{k}^{\ell_{r}}$ in terms of $a_{k}^{\ell_{r}-1}$ is then inserted into the equation for $a_{k}^{\ell_{r}-1}$, obtaining

$$
\left(-i \omega+\nu_{0}\left(\ell_{r}-1\right)+\frac{k^{2}}{\nu_{0}}\right) a_{k}^{\ell_{r}-1}=-i k \sqrt{\ell_{r}-1} a_{k}^{\ell_{r}-2}
$$

We also note that $a_{k}^{\ell_{r}}$ does not appear in the other equations for $a_{k}^{0}, a_{k}^{1}, \ldots, a_{k}^{\ell_{r}-2}$ which remain unchanged. This procedure can be easily iterated and leads to a closed set of equations in which only the collision frequency in the last equation is renormalized

$$
\left(-i \omega+\tilde{\nu}\left[\ell_{c}\right] \ell_{c}\right) a_{k}^{\ell_{c}}+i k \sqrt{\ell_{c}} a_{k}^{\ell_{c}-1}=0
$$

where for $\ell<\ell_{c}$, Eqs. (3)-(5) still apply. The "cut-off" index $\ell_{c}$ determines where the iterative procedure is stopped. The truncation procedure involves a starting point $\ell_{r}$ and a stopping point $\ell_{c}, \ell_{c}<\ell_{r}$, where coefficients are sequentially removed $\ell_{r}, \ell_{r}-1, \ell_{r}-2, \ldots, \ell_{c}-1$. The validity of the truncation requires $\omega \ll \tilde{\nu}\left[\ell_{c}\right] \ell_{c}$, which in turn sets a lower bounds on the value of $\ell_{c}$. The equation for the renormalized coefficient is given by the following recursion

$$
\tilde{\nu}[\ell-1]=\nu_{0}+\frac{k^{2}}{\tilde{\nu}[\ell](\ell-1)}, \quad \tilde{\nu}\left[\ell_{r}\right]=\nu_{0}
$$

We have assumed that $a_{k}^{\ell}$ with $\ell>\ell_{r}$ are negligible, which implies that $a_{k}^{\ell_{r}}$ should also be small and have a weak influence on $a_{k}^{\ell_{r}-1}$. Thus, $\ell_{r}$ is chosen such that $\tilde{\nu}_{\ell_{r}-1} \approx \nu_{0}$ which requires: $\ell_{r}>k^{2} / \nu_{0}^{2}$. Also, assuming $\ell_{c} \gg 1$, so that $\ell_{c}-1 \approx \ell_{c}$ and $\tilde{\nu}\left[\ell_{c}-1\right] \approx \tilde{\nu}\left[\ell_{c}\right]$ we obtain:

$$
\tilde{\nu}\left[\ell_{c}\right]=\frac{\nu_{0}+\sqrt{\nu_{0}^{2}+\frac{4 k^{2}}{\ell_{c}}}}{2} .
$$

If $\ell_{c} \ll k^{2} / \nu_{0}^{2}<\ell_{r}$, the following approximation is valid

$$
\tilde{\nu}\left[\ell_{c}\right]=\frac{|k|}{\sqrt{\ell_{c}}}
$$

which is the $\nu_{0} \rightarrow 0$, but $\nu_{0}$ finite limit, and we note that it is independent of $\nu_{0}$.

Figure 1 shows a comparison of (a) the naive truncation of simply setting $a_{k}^{\ell_{c}}=0$ with $\ell_{c}=8$, (b) the new truncation with $\ell_{c}=8$, and (c) the new truncation with $\ell_{c}=256$. It is seen that there is fairly good agreement at early times between the large $\ell_{c}$ result and the $\ell_{c}=8$ case with the renormalized collision term. Some recurrence is observed, but it is relatively small. For this example, $\nu_{0}=0.005$ and $k=0.5$.

At this point it is important to compare the present truncation with similar iteration schemes used in the study of hydrodynamic turbulence. Let us temporarily set $E=0$ for simplicity (this is a convenient approximation here since 
this term does not influence linear truncation scheme). Fourier transforming $f$ in both $x$ and $v$ using $k$ and $y$ as the conjugate variables, the linear equation may then be rewritten as follows:

$$
\partial_{t} f+\left(k+\nu_{0} y\right) \partial_{y} f+\nu_{0} y^{2} f=0
$$

The first and last term are identical to the linear propagator in the NS problem, the middle term convects perturbations to larger $y$, which is finer velocity scale. So, the interesting question arises, is there any similarity between transfer to shorter (dissipative) scales in NS turbulence, and transfer to finer velocity scales where collisional dissipation dominates in the plasma problem? Dropping the $\nu_{0} y$ term (which should be valid for $k v_{t e} \gg \nu_{0}$ ) we obtain $\partial_{t} f+k \partial_{y} f+\nu_{0} y^{2} f=0$. If the initial condition is noted $f_{0}(y)$, the solution at time $t$ is given by:

$$
f(y, t)=f_{0}(y-k t) \exp \left(-\frac{\nu_{0} y^{3}}{3 k}\right) .
$$

For example, if the initial distribution is peaked around zero $\left(f_{0}=\delta(y)\right)$, the solution at time $t$ will be $f(y)=$ $\delta(y-k t) \exp \left(-\nu_{0} k^{2} t^{3} / 3\right)$, which clearly demonstrates wave propagation to large $y$, along with damping. Therefore, the transfer to finer velocity scales in the plasma problem we discuss here is essentially a linear effect while transfer to finer spatial scales in the NS turbulence is due to nonlinear coupling. As a consequence, the renormalization of the collision coefficient $\nu_{0}$ appears even in the linearized equations. In contrast, the viscosity is not renormalized in the NS equations when the nonlinear term is neglected (very small Reynolds number).

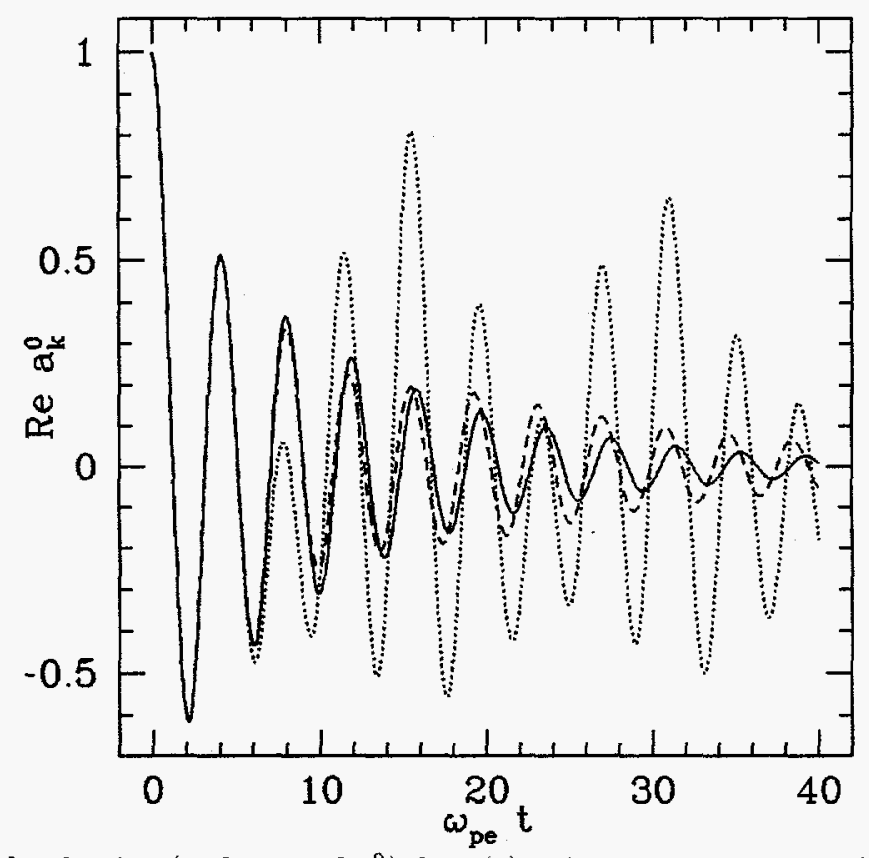

FIG. 1. Time evolution of the density (real part of $a_{k}^{0}$ ) for: (a) naive truncation with $\ell_{c}=8$, short dashed line, (b) new truncation with $\ell_{c}=8$, long dashed line, and (c) new truncation with $\ell_{c}=256$, solid line.

Taking the $\nu_{0} \rightarrow 0$, but $\nu_{0}$ finite, and keeping only a few coefficients the resulting equations reduce to a form similar to the Landau-fluid equations [14]. Multiplying Eq. (2) by $h_{n}(v)$ and integrating over $v$, one finds, $n=(2 \pi)^{1 / 4} a^{0}$, $u=(2 \pi)^{1 / 4} a^{1}$, and $T=\sqrt{2}(2 \pi)^{1 / 4} a^{2}$ [15], using the definitions of $n, u$, and $T$ in Ref. [14], and keeping only linear quantities, from Eqs. (4) and (5), the continuity and momentum equations can be written as

$$
\begin{aligned}
& -i \omega n_{k}+i k u_{k}=0, \\
& -i \omega u_{k}+i k\left(n_{k}+T_{k}\right)+E_{k}=0 .
\end{aligned}
$$

Using Eqs. (8) and (11) with $\ell_{c}=3$, we obtain 


$$
-i \omega T_{k}+2 i k u_{k}+\sqrt{3}|k| T_{k}=0
$$

This result is very similar to the 3 -moment Landau-fluid closure $[14,16]$ where the factor in the dissipative term of Eq. (16) is $\sqrt{8 / \pi}$ versus $\sqrt{3}$ obtained here. Such good agreement may be fortuitous since $\ell_{c}=3$, where as, the $\ell_{c} \gg 1$ limit has been taken. However, good agreement would be expected for larger $\ell_{c}$ (many fluid moments) where the truncation is valid.

The problem of transforming the linear truncation back to real $(x, v)$ space is complicated by the fact that the renormalized term only appears in the last equation for $a_{k}^{\ell_{c}}$. However, we note that a dissipative term of the form

$$
D_{r}[f] \equiv \frac{1}{\ell_{c}^{2 r+1}}\left[\partial_{v}\left(\partial_{v}+v\right)\right]^{2 r+1} f(v)
$$

in velocity space, appears as $-\left(\ell / \ell_{c}\right)^{2 r+1} a_{\ell}$ in the Hermite expansion [9]. Let us consider the large $(2 r+1)$ limit of this expression. The damping term Eq. (17) is then approximately zero for $\ell<\ell_{c}$ so it will not influence the coarse velocity scale. However, it will rapidly damp fine velocity scale when $\ell>\ell_{c}$ which is important for consistency with the discrete truncation. Finally, for $\ell=\ell_{c}$ the damping term will coincide with the renormalized collision frequency if it is multiplied by the factor $\tilde{\nu}\left[\ell_{c}\right] \ell_{c}$. To determine $\ell_{c}$, we relate it to the finest velocity scale, $\lambda$ we wish to resolve

$$
\frac{1}{\lambda}=\frac{1}{h_{\ell_{c}}(v)} \frac{d h_{\ell_{c}}(v)}{d v} \approx \sqrt{\ell_{c}}
$$

assuming $\ell_{c} \gg 1$. The factor $\tilde{\nu}\left[\ell_{c}\right] \ell_{c}$ can then be approximated by $|k| \lambda^{-1}$ using Eqs. (11) and (18). The resulting equation in $(k, v)$ space is

$$
\begin{array}{r}
\partial_{t} \bar{f}_{k}+i k v \bar{f}_{k}-\sum_{k^{\prime}} E_{k^{\prime}} \bar{f}_{k-k^{\prime}}=\nu_{0} \partial_{v}\left(v \bar{f}_{k}+\partial_{u} \bar{f}_{k}\right) \\
+|k| \lambda^{4 r+1}\left[\partial_{v}\left(\partial_{v}+v\right)\right]^{2 r+1} \bar{f}_{k}+N_{2}(k, v)
\end{array}
$$

where the bar signifies $f$ with velocity scales finer than $\lambda$ removed. For example, in a Vlasov-type simulation, $\lambda$ could be determined by the coarseness of the velocity space grid, i.e. $\lambda \approx \Delta v$. The $E \partial_{v} f$ nonlinearity is included and a second nonlinear term appears in the renormalized equations as will be discussed below. Setting $r=0$ and neglecting $N_{2}$, we obtain an equation of the same form as the original, Eq. (1)

$$
\partial_{t} \bar{f}+i k v \bar{f}_{k}-\sum_{k^{\prime}} E_{k^{\prime}} \bar{f}_{k-k^{\prime}}=\bar{\nu} \partial_{v}\left(v \bar{f}_{k}+\partial_{v} \bar{f}_{k}\right)
$$

where $\bar{\nu}=\nu_{0}+|k| \lambda$. However, we are cautious of the validity of Eq. (20) because the diffusive operator $D_{r}$ more accurately represents the truncation for $r>0$.

In order to transform back to real space, we use the following relation $\sum_{k}-i \operatorname{sign}(k) g_{k}=I[g]$ where

$$
I[g]=\frac{1}{\pi} \int_{-\infty}^{+\infty} d y \frac{g(x-y)-g(x+y)}{y}
$$

and note that $|k|=k \operatorname{sign}(k)$. We then obtain the following equation for $\vec{f}(x, v, t)$

$$
\begin{array}{r}
\partial_{t} \bar{f}+v \partial_{x} \bar{f}-E \partial_{v} \bar{f}=\nu_{0} \partial_{v}\left(\partial_{v}+v\right) \bar{f} \\
+\lambda^{-1} D_{r}\left[\partial_{x} I[\bar{f}]\right]+N_{2}(x, v)
\end{array}
$$

The fine velocity scale cut-off, $\lambda$ determines $\ell_{c}$ in the expression for the operator $D_{r}$, Eq. (17), using the relation $\ell_{c}=1 / \lambda^{2}$. Also, the larger the value of $(2 r+1)$, the more accurately the operator $D_{r}$ represents the discrete truncation. The first term on the second line of Eq. (22) is dissipative and models transfer from coarse to fine velocity scale by phase mixing. The second nonlinearity, $N_{2}$ will be calculated below. 
We have successfully applied the truncation procedure to the linearized Boltzmann equation, Eq (1). However, this technique is not restricted to linear problems. In order to illustrate this point, we briefly discuss the renormalization procedure including the $E \partial_{v} f$ nonlinearity. For $\ell>1$, the equation including the nonlinear term is

$$
\begin{gathered}
\left(-i \omega+\nu_{0} \ell\right) a_{k}^{\ell}+i k\left\{\sqrt{\ell} a_{k}^{\ell-1}+\sqrt{\ell+1} a_{k}^{\ell+1}\right\} \\
+\sqrt{\ell} \sum_{k^{\prime}} E_{k^{\prime}} a_{k-k^{\prime}}^{\ell-1}=0 .
\end{gathered}
$$

Here again, we assume $a_{k}^{\ell} \approx 0$ for $\ell>\ell_{r}$ and consider the small frequency limit. The expression for $a_{k}^{\ell_{c}}$, in terms of $a_{k}^{\ell_{c}-1}$, is obtained from Eq. (23) and then is inserted in the equation for $a_{k}^{\ell-1}$ which results in

$$
\begin{aligned}
& \left\{-i \omega+\tilde{\nu}\left[k, \ell_{r}-1\right]\left(\ell_{r}-1\right)\right\} a_{k}^{\ell_{r}-1} \\
& +i k \sqrt{\ell_{r}-1} a_{k}^{\ell_{r}-2}+\sqrt{\ell_{r}-1} \sum_{k^{\prime}} E_{k^{\prime}} a_{k-k^{\prime}}^{\ell_{r}-2} \\
& -i \sum_{k^{\prime}} \gamma\left[\ell_{r}-1\right] E_{k^{\prime}} a_{k-k^{\prime}}^{\ell_{r}-1}=0,
\end{aligned}
$$

where $\gamma\left[\ell_{r}\right]=0$ and $\gamma\left[\ell_{r}-1\right]=k / \nu_{0}$. Next, we assume that the nonlinear term is $O(\epsilon)$ smaller than the linear terms and treat the nonlinearity perturbatively by expanding in series of $\epsilon$. The expansion parameter $\epsilon$ relates the amplitude of the electric field. At this point we stress a major difference between the present approach and renormalization theories of hydrodynamic turbulence $[12,13]$. In these theories, the nonlinearity is also treated perturbatively. However, the limit $\epsilon \rightarrow 0$ is singular in the sense that turbulence disappears for very small $\epsilon$. We have already pointed out that hydrodynamic turbulence is essentially a nonlinear problem which makes the perturbative treatment of the nonlinear convective term in the NS equation more difficult to justify and its range of validity not well known. Here, the situation is very different, where for $\epsilon \rightarrow 0$, we simply recover the linear problem discussed before. Hence, there will be some range of validity for small $\epsilon$. By using this expansion, we obtain

$$
\begin{aligned}
a_{k}^{\ell_{r}-1} \approx- & \frac{1}{\tilde{\nu}\left[\ell_{r}-1\right] \sqrt{\ell_{r}-1}}\left\{i k a_{k}^{\ell_{r}-2}+\sum_{k^{\prime}} E_{k^{\prime}} \times\right. \\
& \left.\left(1-\frac{\gamma\left[\ell_{r}-1\right]\left(k-k^{\prime}\right)}{\tilde{\nu}\left[k-k^{\prime}, \ell_{r}-1\right] \ell_{r}-1}\right) a_{k-k^{\prime}}^{\ell_{r}-2}\right\}
\end{aligned}
$$

This solution can now be inserted into the equation for $a_{k}^{\ell_{r}-2}$ and the procedure can be iterated using the same approach as for linear problem. The last equation for $a_{k}^{\ell_{c}}$ is renormalized by two terms exactly like Eq. (24). The first term is the renormalized collision term and is the same as in the linear case. The second term is characterized by the new renormalized coupling coefficient $\gamma[\ell]$ (with $\ell=\ell_{c}$ ) which is given by

$$
\gamma[\ell-1]=\frac{k}{\tilde{\nu}[k, \ell]}-\gamma[\ell] \frac{k\left(k-k^{\prime}\right)}{\tilde{\nu}[k, \ell] \tilde{\nu}\left[k-k^{\prime}, \ell\right] \ell}
$$

Eqs. (9) and (26) are recursive relations starting at the resolution index $\ell_{r}$ and working down to the cut-off index $\ell_{c}$, $\ell_{c}<\ell_{r}$. For $\ell_{c} \ll \ell_{r}, \gamma\left[\ell_{c}\right]$ is well approximated by

$$
\gamma\left[\ell_{c}\right]=\left(s_{1}+s_{2}\right) \frac{\sqrt{\ell_{c}}}{4}+s_{1}\left(s_{1}-s_{2}\right) \frac{k\left(k-k^{\prime}\right)}{\nu_{0} k^{\prime}},
$$

where $s_{1}=\operatorname{sign}(k)$ and $s_{2}=\operatorname{sign}\left(k-k^{\prime}\right)$. Finite collsionality is needed in Eq. (27) for $\gamma$ to be finite for couplings where $k\left(k-k^{\prime}\right)<0$, i.e. coupling from $f_{k-k^{\prime}}$ modes with a sign opposite to $k$. Using the same kind of approximations as for the linear problem, we may now transform back to $(k, v)$ space. Resulting in the following nonlinear term for Eq. (19)

$$
N_{2}(k, v)=i \sum_{k^{\prime}} \gamma\left[\ell_{c}=\frac{1}{\lambda^{2}}, k, k-k^{\prime}\right] E_{k^{\prime}} D_{r} \bar{f}_{k-k^{\prime}}
$$


Transforming back to $(x, v)$ space the additional nonlinearity is given by

$$
\begin{aligned}
N_{2}(x, v)=-D_{r} & {\left[\frac{1}{4 \lambda}(I[E \bar{f}]+E I[\bar{f}])\right.} \\
& \left.+\frac{1}{\nu_{0}} \partial_{x}\left(\phi \partial_{x} \bar{f}+I\left[\phi \partial_{x} I[\bar{f}]\right]\right)\right],
\end{aligned}
$$

where $\phi$ is the electric potential $E=-\partial_{x} \phi$.

We have developed a consistent truncation of a Boltzmann-type equation where the result is a renormalized kinetic equation which is coarse grained in velocity space. In contrast to the hydrodynamic Navier-Stokes equation, the plasma Boltzmann equation is renormalized even with the nonlinear term neglected. In the linear case, the result is a renormalization of the collision frequency and modification of the collision operator. We have also shown that the procedure can be applied to nonlinear problems by perturbative expansion, using the $E \partial_{v} f$ as an example, resulting in an additional mode coupling term. Here, we illustrated the procedure on a simple one dimensional unmagnetized electrostatic Boltzmann equation. Application of this truncation procedure to drift-kinetic and gyrokinetic equations with weak collisionality will be reported in the future.

We thank G.W. Hammett, W.W. Lee, M. Schoucri, and S.A. Smith. Work (SEP) supported by the U.S. DOE Contract No. DE-AC02-76-CHO-3073 and (DC) the National Fund for the Scientific Research (Belgium).

[1] F.C. Grant and M.C. Feix, Phys. Fluids, 10696 (1967).

[2] F.C. Grant and M.C. Feix, Phys. Fluids, 101356 (1967).

[3] T. Armstrong Phys. Fluids, 101269 (1967).

[4] T.P. Armstrong, et al., in "Methods of Computational Physics," B. Alder, et al., editors, 929 (1970), Academic Press, NY.

[5] G. Joyce, G. Knorr and H.K. Meier, J. Comput. Phys., 853 (1970).

[6] G. Knorr, J. Comput. Phys., 13165 (1973).

[7] G. Knorr and M. Shoucri, J. Comput. Phys., 141 (1974).

[8] M. Shoucri and G. Knorr, J. Comput. Phys., 1484 (1974).

[9] M. Shoucri and R. Gangne, J. Comput. Phys., 21238 (1976).

[10] G. W. Hammett, M. A. Beer, W. Dorland, S. C. Cowley, and S. A. Smith, Plasma Phys. Control. Fusion 35, 973 (1993).

[11] D. Forster, D. Nelson, and M. Stephen, Phys. Rev. A 16, 732 (1977).

[12] J. Fournier and U. Frisch, Phys. Rev. A 28, 1000 (1983).

[13] V. Yakhot and S. Orszag, J. Sci. Comp. 1, 3 (1986).

[14] G. W. Hammett and F.W. Perkins, Phys. Rev. Lett. 643019 (1990).

[15] S.A. Smith and G. W. Hammett, "Some nonlinear aspects of fluid moment closures that model landau damping," to be published, private communication (1994).

[16] W. Dorland and G. W. Hammett, Phys. Fluids B 5812 (1993). 
Dr. F. Paoloni, Univ. of Wollongong, AUSTRALIA

Prof. R.C. Cross, Univ. of Sydney, AUSTRALIA

Plasma Research Lab., Australian Nat. Univ., AUSTRALIA

Prol. I.R. Jones, Flinders Univ, AUSTRALIA

Prof. F. Cap, Inst. for Theoretical Physics, AUSTRIA

Prof. M. Heindler, Institut für Theoretische Physik, AUSTRIA

Prof. M. Goossens, Astronomisch Instituut, BELGIUM

Ecole Royale Militaire, Lab. de Phy. Plasmas, BELGJUM

Commission-European, DG. XII-Fusion Prog., BELGIUM

Prof. R. Bouciqué, Rijksuniversiteit Gent, BELGIUM

Dr. P.H. Sakanaka, Instituto Fisica, BRAZIL

Prof. Dr. I.C. Nascimento, Instituto Fisica, Sao Paulo, BRAZIL Instituto Nacional De Pesquisas Espaciais-INPE, BRAZIL Documents Office, Atomic Energy of Canada Ltd., CANADA Ms. M. Morin, CCFWTokamak de Varennes, CANADA Dr. M.P. Bachynski, MPB Technologies, Inc., CANADA

Dr. H.M. Skarsgard, Univ. of Saskatchewan, CANADA

Prof. J. Teichmann, Univ. of Montreal, CANADA

Prof. S.R. Sreenivasan, Univ, of Calgary, CANADA

Prof. R. Marchand, INRS-Energie et Materiaux, CANADA

Dr. R. Bolton, Centre canadien de fusion magnétique, CANADA

Dr. C.R. James, Univ. of Alberta, CANADA

Dr. P. Lukác, Komenského Universzita, CZECHO-SLOVAKIA

The Librarian, Culham Laboratory, ENGLAND

Library, R61, Rutherford Appleton Laboratory, ENGLAND

Mrs. S.A. Hutchinson, JET Library, ENGLAND

Dr. S.C. Shama, Univ. of South Pacific, FIJI ISLANDS

P. Mähönen, Univ. of Helsinki, FINLAND

Prof. M.N. Bussac, Ecole Polytechnique, FRANCE

C. Mouttet, Lab. de Physique des Milieux lonisés, FRANCE

J. Radet, CEN/CADARACHE - Bat 506, FRANCE

Prof. E. Economou, Univ. of Crete, GREECE

Ms. C. Rinni, Univ. of loannina, GREECE

Preprint Library, Hungarian Academy of Sci., HUNGARY

Dr. B. DasGupta, Saha Inst. of Nuclear Physics, INDIA

Dr. P. Kaw, Inst. for Plasma Research, INDIA

Dr. P. Rosenau, Israel Inst. of Technology, ISRAEL

Librarian, International Center for Theo Physics, ITALY

Miss C. De Palo, Associazione EURATOM-ENEA, ITALY

Dr. G. Grosso, Istituto di Fisica del Plasma, ITALY

Prof. G. Rostangni, Istituto Gas Ionizzati Del Cnr, ITALY
Dr. H. Yamato, Toshiba Res \& Devel Center, JAPAN

Prof. I. Kawakami, Hiroshima Univ., JAPAN

Prof. K. Nishikawa, Hiroshima Univ., JAPAN

Librarian, Naka Fusion Research Establishment, JAERI, JAPAN

Director, Japan Atomic Energy Research Inst., JAPAN

Prof. S. Itoh, Kyushu Univ., JAPAN

Research Info. Ctr., National Instit. for Fusion Science, JAPAN

Prof. S. Tanaka, Kyoto Univ., JAPAN

Library, Kyoto Univ., JAPAN

Prof. N. Inoue, Univ. of Tokyo, JAPAN

Secretary, Plasma Section, Electrotechnical Lab., JAPAN

Dr. O. Mitarai, Kumamoto Inst. of Technology, JAPAN

Dr. G.S. Lee, Korea Basic Sci. Ctr., KOREA

J. Hyeon-Sook, Korea Atomic Energy Research Inst., KOREA

D.I. Choi, The Korea Adv. Inst. of Sci. \& Tech., KOREA

Leandro Melendez Lugo, Inst. Nac'l. de Inves. Nud, MEXICO

Prof. B.S. Liley, Univ, of Waikato, NEW ZEALAND

Inst of Physics, Chinese Acad Sci PEOPLE'S REP. OF CHINA

Library, Inst. of Plasma Physics, PEOPLE'S REP. OF CHINA

Tsinghua Univ. Library, PEOPLE'S REPUBLIC OF CHINA

Z. Li, S.W. Inst Physics, PEOPLE'S REPUBLIC OF CHINA

Prof. J.A.C. Cabral, Instituto Superior Tecnico, PORTUGAL

Prof. M.A. Hellberg, Univ. of Natal, S. AFRICA

Prof. D.E. Kim, Pohang Inst. of Sci. \& Tech., SO. KOREA

Prof. C.I.E.M.A.T, Fusion Division Library, SPAIN

Dr. L. Stenflo, Univ. of UMEA, SWEDEN

Library, Royal Inst. of Technology, SWEDEN

Prof. H. Wilhelmson, Chalmers Univ. of Tech., SWEDEN

Centre Phys. Des Plasmas, Ecole Polytech, SWITZERLAND

Bibliotheek, Inst. Voor Plasma-Fysica, THE NETHERLANDS

Asst. Prof. Dr. S. Cakir, Middle East Tech. Univ., TURKEY

Dr. V.A. Glukhikh,Sci. Res. Inst. Electrophys.I Apparatus, USSR

Dr. D.D. Ryutov, Siberian Branch of Academy of Sci., USSR

Dr. G.A. Eliseev, I.V. Kurchatov Inst., USSR

Librarian, The Ukr.SSR Academy of Sciences, USSR

Dr. L.M. Korrizhnykh, Inst. of General Physics, USSR

Kemforschungsanlage GmbH, Zentralbibliothek, W. GERMANY

Bibliothek, Inst. Für Plasmaforschung, W. GERMANY

Prof. K. Schindler, Ruhr-Universitát Eochum, W. GERMANY

Dr. F. Wagner, (ASDEX), Max-Planck-Institut, W. GERMANY

Librarian, Max-Planck-Institut, W. GERMANY 